\title{
Extracellular Matrix Gene Expression Increases Preferentially in Rat Lipocytes and Sinusoidal Endothelial Cells during Hepatic Fibrosis In Vivo
}

Jacquelyn J. Maher and Richard F. McGuire

Liver Core Center and Department of Medicine, University of California, San Francisco, California 94110

\begin{abstract}
Whether parenchymal or nonparenchymal liver cells play a predominant role in the pathophysiology of hepatic fibrosis has not been firmly established in vivo. We have addressed this question by quantitating the relative abundance of specific mRNAs for collagen types I, III, and IV, and laminin in purified populations of hepatocytes, sinusoidal endothelial cells, and lipocytes from normal and fibrotic rat liver. In normal liver, type I collagen gene expression was minimal in all cell types; mRNA for types III and IV collagen were apparent in endothelial cells and lipocytes, but not in hepatocytes. Laminin mRNA was present in all cell types. Induction of fibrogenesis by either bile duct ligation or carbon tetrachloride administration was associated with a substantial increase in mRNA for types I and III collagen in nonparenchymal cells. Lipocytes from fibrotic animals exhibited a $>30$-fold increase in type I collagen mRNA relative to normal lipocytes, and $>40$-fold relative to hepatocytes. Type III collagen mRNA reached 5 times that in normal lipocytes and $>120$ times that in hepatocytes. Endothelial cells exhibited an isolated increase in type I collagen mRNA, reaching five times that in normal liver. Type IV collagen and laminin gene expression were not significantly increased in nonparenchymal cells during fibrogenesis; in fact, mRNA for type IV collagen and laminin decreased by up to $\mathbf{5 0} \%$ in endothelial cells. Despite the pronounced changes that occurred in matrix gene expression in nonparenchymal cells during fibrogenesis, no change was noted in hepatocytes. We conclude that nonparenchymal liver cells, particularly lipocytes, are important effectors of hepatic fibrosis in vivo. ( $J$. Clin. Invest. 1990. 86:1641-1648.) Key words: collagen • laminin • liver • cirrhosis $\bullet$ hepatocytes
\end{abstract}

\section{Introduction}

Liver fibrosis, regardless of its cause, is characterized by a marked accumulation of extracellular matrix material within the perisinusoidal space of Disse. Interstitial collagens (types I and III) comprise the bulk of the newly deposited matrix (1-3), although basement membrane (type IV) collagen and noncollagenous glycoproteins, such as laminin and fibronectin, also

A portion of this work has been published in abstract form in 1989 (Hepatology [Baltimore] 10:629a).

Address reprint requests to Dr. J. Maher, Liver Center Laboratory, San Francisco General Hospital, Bldg. 40 Rm. 4102, 1001 Potrero Ave., San Francisco, CA 94110. 1990.

Received for publication 13 April 1990 and in revised form 28 June

J. Clin. Invest.

(c) The American Society for Clinical Investigation, Inc.

$0021-9738 / 90 / 11 / 1641 / 08 \quad \$ 2.00$

Volume 86, November 1990, 1641-1648 contribute (4). Numerous efforts have been made to identify the cellular sources of extracellular matrix proteins in intact liver. Immunohistochemical studies suggest that in normal liver, extracellular matrix proteins are present in both parenchymal and nonparenchymal liver cells (5-7). After carbon tetrachloride administration, type IV collagen and laminin are localized in sinusoidal endothelial cells and lipocytes (Ito cells, fat-storing cells), whereas types I and III collagen reportedly are present in hepatocytes $(8,9)$. These observations suggest that parenchymal liver cells play a central role in the production of interstitial collagen that accompanies hepatic fibrosis. In vivo labeling studies also suggest that the majority of collagen extracted from liver after carbon tetrachloride administration derives from hepatocytes (10). Other experiments, however, have led to a different conclusion, that hepatic extracellular matrix synthesis is largely a function of mesenchymal liver cells. Experiments examining fibrotic animal and human liver by in situ hybridization demonstrate that messenger RNAs for collagen types I, III, and IV, and laminin are concentrated in perisinusoidal cells (11-13). Based on these conflicting results, debate still exists regarding which cell population is the major contributor to hepatic fibrosis.

Although immunohistochemistry and in situ hybridization are useful for examining the origin of extracellular matrix in intact tissue, they provide only qualitative information, and at the level of light microscopy cannot pinpoint the exact source of extracellular matrix protein or mRNA. These limitations can be circumvented in part by adopting a cell culture approach, which permits quantitative measurements of matrix synthesis in purified populations of liver cells. Studies in culture favor nonparenchymal cells as the primary source of extracellular matrix in liver, demonstrating that lipocytes far exceed hepatocytes in collagen synthesis on a per-cell basis (14-16). Even these findings must be interpreted with caution, however, in view of evidence that cells in culture are susceptible to changes in collagen phenotype (17-19). An ideal method for defining the cellular source(s) of extracellular matrix in liver in vivo must incorporate the advantages of both approaches above, providing a means to quantitate matrix within individual liver cell populations without imposing the influence of a foreign (culture) environment. Clayton and Darnell (20) have shown that the mRNA profile of liver cells immediately following isolation from the intact organ recapitulates that in vivo. In the current study, this method has been applied to parenchymal and nonparenchymal liver cells from normal and fibrotic liver, to assess expression of various extracellular matrix genes in vivo. We demonstrate in two mechanistically distinct models of hepatic fibrosis in the rat, that extracellular matrix gene expression increases exclusively in mesenchymal liver cells. Furthermore, expression of the gene for type I collagen is enhanced to the greatest extent in hepatic lipocytes. As type I collagen is the predominant constituent of the fibrotic hepatic matrix, these results suggest that lipocytes 
figure prominently in the pathophysiology of hepatic fibrosis in vivo.

\section{Methods}

\section{Materials}

[Alpha $\left.{ }^{32} \mathrm{P}\right]$ cytidine-5'-triphosphate $\left(\left[\alpha-{ }^{32} \mathrm{P}\right] \mathrm{CTP}\right)(>800 \mathrm{Ci} / \mathrm{mmol})$ (SP6 grade) was purchased from Amersham Corp., Arlington Heights, IL. Pronase and collagenase B were purchased from Boehringer Mannheim Biochemicals, Indianapolis, IN; DNase I was from Calbiochem-Behring Corp., La Jolla, CA. DME and Ham's F-12 media were obtained from Flow Laboratories, Inc., McLean, VA; Eagle's MEM without calcium (MEM-E) was prepared in the laboratory using amino acids from Sigma Chemical Co., St. Louis, MO. Larex ${ }^{\circledR}$ (arabinogalactan) was obtained from Larex International, Tacoma, WA. Formalinfixed $S$. aureus was purchased from Zymed Laboratories, Burlingame, CA. Human acetoacetylated low-density lipoprotein, conjugated with 3,3'-dioctadecyl indocarbocyanine (DiI), was generously supplied by Dr. R. Pitas, Gladstone Foundation, San Francisco, CA. Guanidine thiocyanate was purchased from Fluka Chemical Corp., Ronkonkoma, NY. Cesium chloride (molecular biology grade) and formamide were from International Biotechnologies, Inc., New Haven, CT. Polyacrylamide and agarose (molecular biology grade) were obtained from Bio-Rad Laboratories, Richmond, CA; ultrapure urea was from Bethesda Research Laboratories, Gaithersburg, MD. Restriction enzymes and RNase T2 were purchased from Bethesda Research Laboratories. SP6 and T7 RNA polymerases were from Promega Biotec, Madison, WI. Polyclonal rabbit anti-chicken desmin was from Dako Corp., Santa Barbara, CA.

\section{Procedures}

Animal models of fibrogenesis. Mild hepatic fibrosis was induced in male Sprague-Dawley rats by either bile duct ligation (BDL) ${ }^{1}$ or carbon tetrachloride administration (CCL). Bile duct ligation and scission were performed under ether anesthesia. Animals were maintained postoperatively on food and water ad lib. and killed for liver cell isolation on postoperative day 5 . Carbon tetrachloride $(50 \% \mathrm{vol} / \mathrm{vol}$ in corn oil, $0.1 \mathrm{~cm}^{3} / 100 \mathrm{~g}$ body wt i.p.) was administered to unanesthetized rats on days 1,4 , and 7 . Injected animals were allowed $7 \mathrm{~d}$ to recover from acute hepatic injury, and killed for liver cell isolation on day 14. Normal Sprague-Dawley rats served as controls for both models of fibrosis.

Histology. Rat livers subjected to experimental injury as described above were perfusion-fixed with $4 \%$ paraformaldehyde in $0.1 \mathrm{M}$ phosphate (pH 7.4) and embedded in paraffin. Cut sections were stained with Gordon and Sweet's reticulin.

Liver cell isolation and purification. Hepatocytes were isolated from control and experimental animals by collagenase perfusion and centrifugal elutriation, as previously described $(16,21)$. Sinusoidal endothelial cells and lipocytes were isolated by in situ perfusion with collagenase and pronase; the two cell populations were separated by density gradient centrifugation through Larex ${ }^{\oplus}$, as described (22). Lipocytes, which remained at the top two interfaces, were collected and washed twice in culture medium followed by centrifugation at $500 \mathrm{~g}$ to remove hepatocyte debris. Endothelial cells, collected from the bottom two interfaces of the gradient, were purified further by centrifugal elutriation at $2,500 \mathrm{rpm}$ and a flow rate of $10-18 \mathrm{ml} / \mathrm{min}(\mathrm{J} 2-21$ centrifuge, with JE6-B rotor and Sanderson chamber; Beckman Instruments, Inc., Palo Alto, CA). Purity of all isolates was assessed by direct cell counting, using DiI-conjugated acetoacetylated low-density lipoprotein (21), fluorescein-conjugated $S$. aureus (22), and anti-desmin (23) as markers for endothelial cells, Kupffer cells, and lipocytes, respectively. Lipocytes could also be distinguished by their intrinsic fluorescence under ultraviolet illumination.

1. Abbreviations used in this paper: $\mathrm{BDL}$, bile duct ligation; $\mathrm{CCL}$, carbon tetrachloride.
Probe preparation. cDNAs encoding rat $\alpha_{1}(\mathrm{I})$ collagen $\left(\mathrm{p} \alpha_{1} \mathrm{R} 1\right)$ (24), rat $\alpha_{1}$ (III) collagen (PRGR5) (Makela, J. K., and E. Vuorio, unpublished observations), mouse $\alpha_{1}$ (IV) collagen (pPE123) (25), and mouse laminin $B_{2}$ chain (pPE9) (26) were donated to the laboratory. Restriction fragments of each were subcloned into pGEM ${ }^{\oplus}-4$ (Promega Biotec) to enable synthesis of labeled RNA for hybridization. The greatest sensitivity and specificity in RNase protection studies were obtained with the following subclones: $(a)$ for $\mathrm{p} \alpha_{1} \mathrm{R} 1, \mathrm{a} \sim 340$-bp Pst I-Bam HI fragment from the triple helical domain; $(b)$ for pRGR5, $a \sim 280$-bp Hinc II-Eco RI fragment from the $3^{\prime}$ end of the translated sequence; $(c)$ for pPE123, a 380-bp Ava I-Eco RI fragment at the $3^{\prime}$ end of the translated sequence; $(d)$ for pPE9, a 486-bp Bam HI-Sal I fragment encompassing most of the original cDNA. A $\sim 900-b p$ cDNA for human $\beta$-actin (27), which cross-hybridizes with rat $\beta$-actin, was provided in SP64 by Dr. J. M. Friedman, The Rockefeller University, New York. Radiolabeled probes were generated by transcription with SP6 or T7 polymerase using $\left[\alpha^{-32} \mathrm{P}\right] \mathrm{CTP}(28)$. Specific activity of the transcripts was estimated at $0.5 \times 10^{9} \mathrm{cpm} / \mu \mathrm{g}$.

RNase protection (29). Total RNA was extracted from individual liver cell isolates by lysis in guanidine thiocyanate, followed by centrifugation through cesium chloride (30). All extractions were performed immediately after cell isolation and purification. The integrity of each sample was verified by spectrophotometry $\left(\mathrm{A}_{260} / \mathrm{A}_{280}>1.9\right)$ and agarose gel electrophoresis with ethidium bromide staining before use in molecular hybridization studies. $5 \mu \mathrm{g}$ of total RNA was hybridized in solution with $0.25-0.75 \times 10^{6} \mathrm{cpm}$ of ${ }^{32} \mathrm{P}$-labeled cRNA for $16 \mathrm{~h}$. Hybridization temperature was optimized for each probe, varying between $56^{\circ}$ and $70^{\circ} \mathrm{C}$. The mixture was then treated with T2 RNase to digest unhybridized cellular RNA and probe, followed by phenol/chloroform extraction and ethanol precipitation. The remaining intact hybrids were denatured in electrophoresis buffer containing $80 \%$ formamide, and separated by electrophoresis through a $5 \%$ polyacrylamide-urea gel $(21 \times 40 \mathrm{~cm})$. Dried gels were applied to x-ray film (Kodak X-OMat AR-5) for 1-24 h. Autoradiographic signals were quantitated by scanning densitometry (Hoefer Scientific Instruments, San Francisco, CA).

Statistical analysis. All numerical data represent the mean \pm SEM of individual experiments performed on three separate animals. Results with $P<0.05$ by Mann-Whitney U-test were considered significant.

\section{Results}

To assess the degree of hepatic fibrosis induced by bile duct ligation and carbon tetrachloride administration, livers from both normal rats and those with experimentally induced fibrosis were examined under light microscopy. As shown in Fig. 1, bile duct-ligated animals exhibited a moderate increase in periportal connective tissue relative to normal animals. Animals exposed to three doses of carbon tetrachloride displayed a much less pronounced change in hepatic connective tissue, although central-central bridging was apparent. At these same time points, parenchymal and nonparenchymal cells were isolated for analysis of various extracellular matrix mRNAs. The purity of each cell isolate is indicated in Table I. Hepatocytes exhibited the greatest homogeneity, with $<2 \%$ contamination by lipocytes. Endothelial cells were $95 \%$ pure, containing approximately equal numbers of Kupffer cells and lipocytes. In animals with experimental fibrosis, the proportion of lipocytes in endothelial cell isolates sometimes reached 10\%; this may be due to increased penetration of the density gradient by lipocytes, which release their low-density vitamin A during fibrogenesis $(17,31)$. The lipocyte fraction was $98 \%$ homogeneous, with Kupffer cells being the primary contaminant.

Steady-state levels of mRNA for collagen types I, III, and 

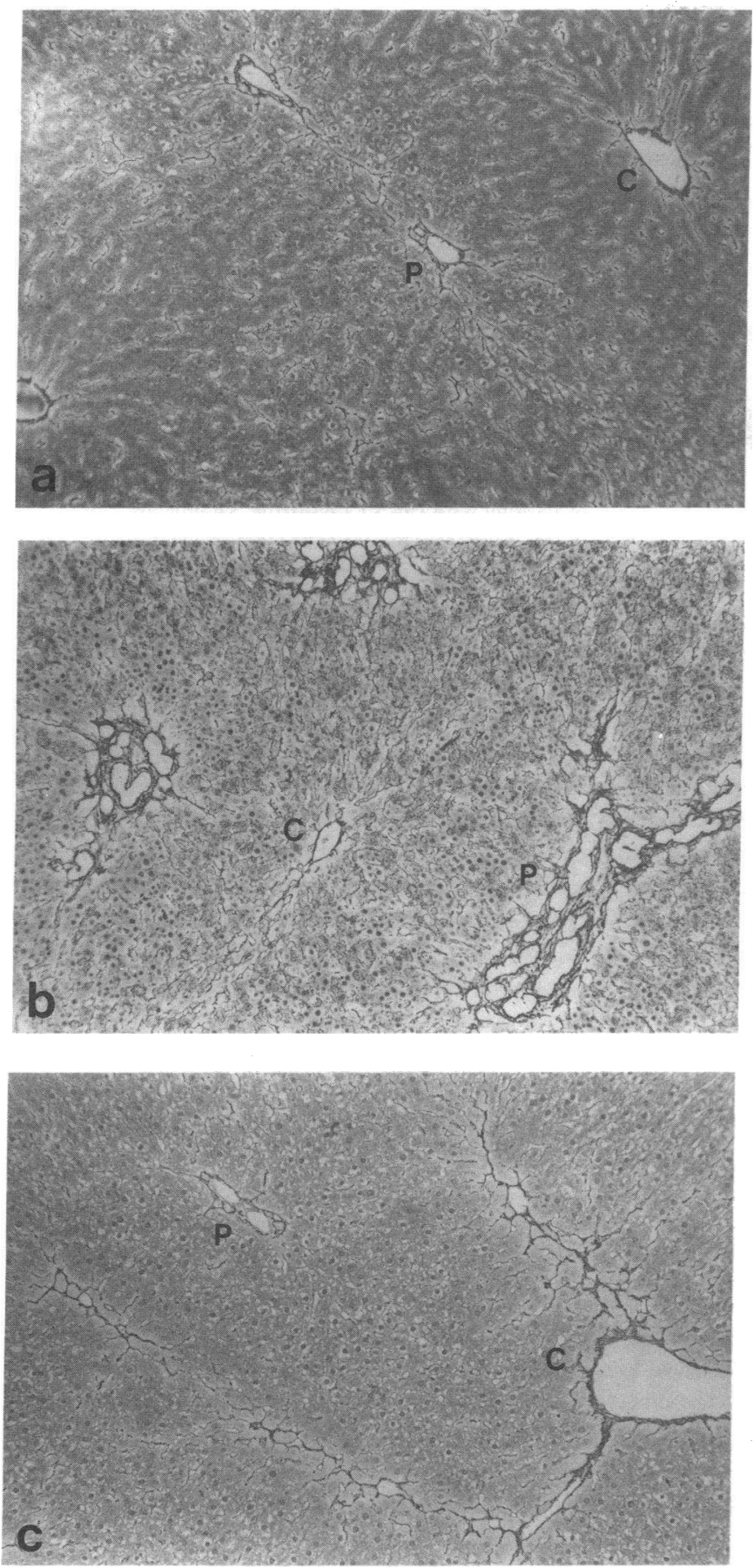

Figure 1. Liver histology. Photomicrographs illustrate the connective tissue distribution in livers from normal $(a)$, bile duct-ligated $(b)$, or CCL-treated $(c)$ rats. Normal liver exhibits only mild connective tissue staining around portal triads $(P)$ and central veins $(C)$. BDL after $5 \mathrm{~d}$ results in an increase in connective tissue in expanded portal regions. CCL after $14 \mathrm{~d}$ produces an increase in pericentral connective tissue, with evidence of central-central bridging. (Gordon and Sweet's reticulin, $10 \times$.)

IV, laminin, and $\beta$-actin were quantitated in liver cell isolates by RNase protection (see Methods). A representative autoradiogram depicting the results for type I collagen is shown in Fig. 2. In normal liver, all three cell types exhibited weak signals for type I collagen mRNA, in comparison to fibroblast
Table I. Purity of Individual Cell Isolates

from Normal and Fibrotic Liver*

$\begin{array}{ll}\text { Hepatocytes } & 98.8 \pm 0.2 \% \\ \text { Endothelial cells } & 95.0 \pm 0.5 \% \\ \text { Lipocytes } & 97.5 \pm 0.4 \%\end{array}$

Values represent mean \pm SEM for a minimum of three separate animals.

* Purity was assessed by direct cell counting, as described in Methods.

controls. After bile duct ligation, type I collagen mRNA was increased in both endothelial cells and lipocytes, but was unchanged in hepatocytes. A similar pattern was observed after carbon tetrachloride administration. Additional experiments measuring mRNA for types III and IV collagen and laminin are illustrated in Fig. 3. In normal liver, type III and IV collagen mRNA were at the limit of detection in normal hepatocytes; a laminin signal was apparent but faint. Endothelial cells and lipocytes, by contrast, exhibited strong hybridization signals for all three matrix genes. Induction of hepatic fibrogenesis by either BDL or CCL was not accompanied by a gross alteration in matrix gene expression by hepatocytes. mRNA levels for type III collagen, type IV collagen, and laminin were all modulated, however, in endothelial cells and lipocytes.

Molecular hybridization experiments were performed in three sets of animals and quantitated by scanning densitometry (Table II; Fig. 4). Signal intensity was normalized to the lowest value in each group (uniformly that in normal hepatocytes), which was given an arbitrary value of 1.0. This allowed direct, quantitative comparisons of matrix gene expression among different cell types and under different experimental conditions. Comparisons are valid only for the experimental data for each individual extracellular matrix gene (e.g., type I collagen in hepatocytes vs. type I collagen in lipocytes); due to differences in probe length and specificity, as well as autoradiographic exposure time, the level of expression of individual extracellular matrix mRNAs for a given cell type cannot be compared (e.g., type I collagen vs. type IV collagen in lipocytes).

Fig. $4 A$ illustrates that in normal liver, steady-state levels of mRNA for type I collagen were similar in both parenchymal and nonparenchymal cells. After bile duct ligation, type I collagen gene expression was increased in both endothelial cells and lipocytes, reaching 7.8 and 49.8 times, respectively, that in normal hepatocytes. Carbon tetrachloride administration resulted in a similar enhancement in type I collagen gene expression, with a 14.8-fold and 43.1-fold increase in endothelial cells and lipocytes, respectively.

Type III collagen mRNA was abundant in nonparenchymal cells from normal liver (Fig. 4 B). Endothelial cells contained 28.5 times and lipocytes 29.0 times the amount of type III collagen mRNA as normal hepatocytes. Type III collagen gene expression remained high in endothelial cells and increased dramatically in lipocytes, following both BDL and CCL injury.

Type IV collagen gene expression in normal liver was dominated by endothelial cells, which contained almost 100 times as much type IV-specific mRNA as normal hepatocytes (Fig. 4 $C)$. Lipocytes also exhibited abundant type IV collagen mRNA, with 35.7 times as much as normal hepatocytes. After 


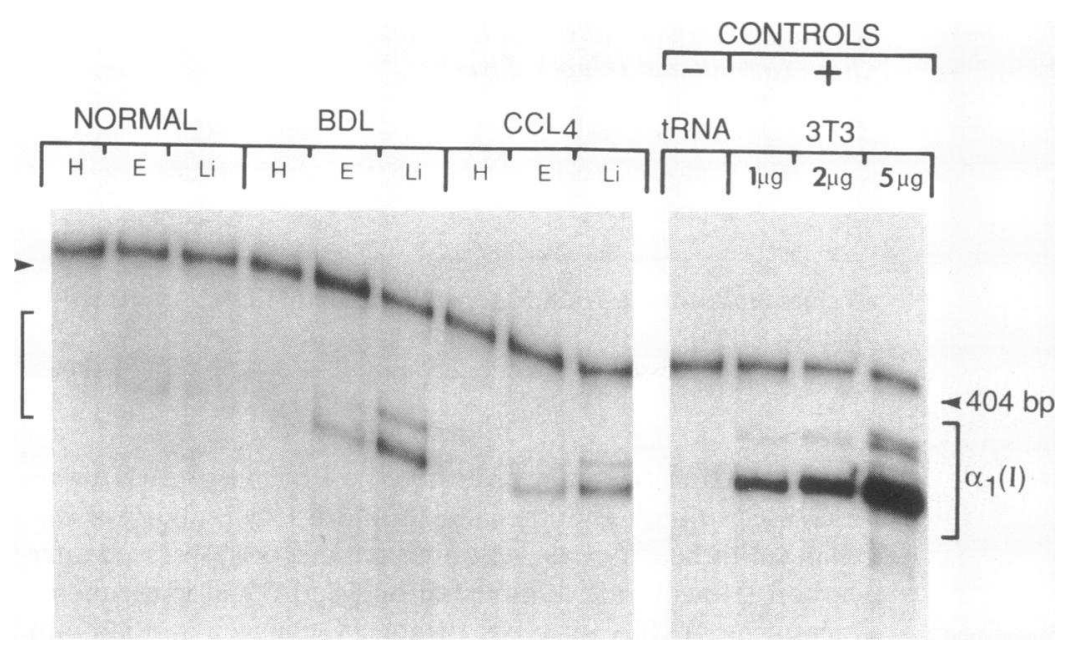

Figure 2. Type I collagen gene expression in individual liver cells as measured by RNase protection (see Methods). Bands represent steady-state levels of type I collagen mRNA in hepatocytes $(H)$, endothelial cells $(E)$, and lipocytes $(L i)$ from normal, bile duct-ligated $(B D L)$, and carbon tetrachloride $(C C L)$-treated animals, respectively. Controls include tRNA from yeast and mRNA from NIH 3T3 fibroblasts, the latter in increasing concentrations to document linearity of the autoradiographic signal. The band above 404 bp represents nonspecific hybridization. Type I collagen mRNA is visible as a doublet at $\sim 340 \mathrm{bp}$, in parallel with the $3 T 3$ controls. Signals are greatest in the nonparenchymal cells from injured livers. hepatic injury with BDL or CCL, type IV collagen gene expression remained high in both cell types; endothelial cells, however, were replaced by lipocytes as the population with the highest levels of type IV collagen mRNA.

Laminin gene expression was also highest in endothelial cells from normal liver, measured at 13.1 times that in normal hepatocytes (Fig. $4 \mathrm{D}$ ). Laminin mRNA in lipocytes was 5.8 times higher than in normal hepatocytes. As in the case of types III and IV collagen, laminin mRNA remained abundant in nonparenchymal cells during fibrogenesis although to different degrees in endothelial cells and lipocytes.

In order to identify the cell-specific changes that occur in extracellular matrix gene expression during hepatic fibrosis in vivo, mRNA levels for collagen types I, III, and IV, and laminin in purified cell populations from experimentally injured livers were compared to those for the corresponding cell type from normal liver (Fig. 5). Viewed in this manner, the data indicate that enhancement of extracellular matrix gene expression during hepatic fibrogenesis was largely a function of hepatic lipocytes. A substantial ( $>30$-fold) increase in type I collagen gene expression was demonstrated in both BDL and CCL-induced fibrogenesis; a smaller, albeit significant, increase in type III collagen gene expression was observed in this cell type. Type IV collagen gene expression was increased in lipocytes following BDL but not CCL in the current experi-

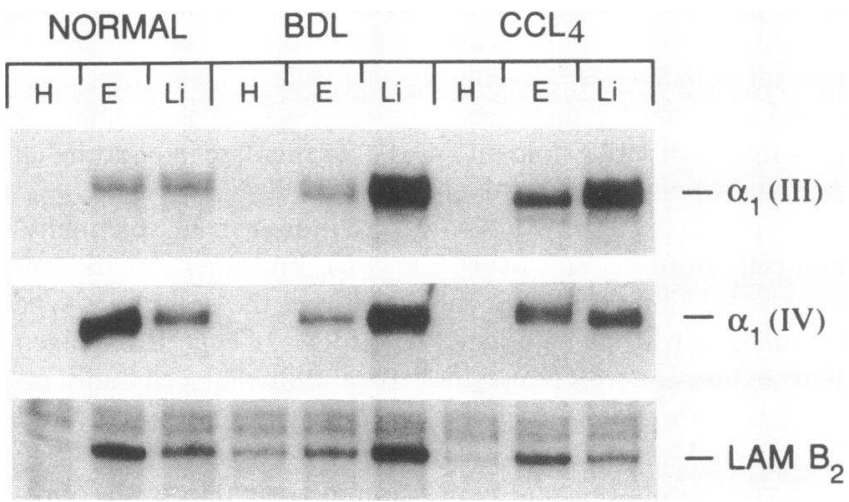

Figure 3. Steady-state levels of mRNA for type III collagen $\left(\alpha_{1}(I I I)\right)$, type IV collagen $\left(\alpha_{1}(I V)\right)$, and laminin $\left(L A M B_{2}\right)$ in cells isolated from normal and fibrotic liver. Abbreviations as in Fig. 2. The strongest hybridization signals are again exhibited by nonparenchymal cells. ments; laminin gene expression was not significantly increased in either model of fibrosis.

Enhancement of extracellular matrix gene expression was less pronounced in endothelial cells during hepatic fibrogenesis (Fig. 5). Type I collagen mRNA was significantly increased in endothelial cells following both BDL and CCL-induced injury; mRNA for type III collagen, however, was unchanged from normal cells, and that for type IV collagen and laminin actually decreased to less than $50 \%$ of normal levels. Hepatocytes were the least responsive of all three cell types to hepatic injury. Neither BDL nor CCL induced a change in steady-state mRNA levels for any of the matrix genes tested. No reductions in extracellular matrix gene expression were observed in hepatocytes following liver injury; it is unlikely, however, that any decrease below normal could be detected in these experiments, given the low hybridization signals in all hepatocyte samples (Figs. 2 and 3).

Table II. Steady-state Levels of Extracellular Matrix mRNA in Liver Cells Isolated from Normal and Injured Rat Liver

\begin{tabular}{lccc}
\hline & Normal & BDL & CCL \\
\hline Collagen I & & & \\
$\quad$ Hepatocytes & $1.0 \pm 0^{*}$ & $1.4 \pm 0.2$ & $0.9 \pm 0.2$ \\
Endothelial cells & $1.9 \pm 0.3$ & $7.8 \pm 2.5^{\ddagger}$ & $14.8 \pm 7.3^{\ddagger}$ \\
$\quad$ Lipocytes & $1.7 \pm 0.3$ & $49.8 \pm 11.8^{\ddagger}$ & $43.1 \pm 13.6^{\ddagger}$ \\
Collagen III & & & \\
$\quad$ Hepatocytes & $1.0 \pm 0^{*}$ & $1.2 \pm 0.2$ & $2.1 \pm 0.9$ \\
Endothelial cells & $28.5 \pm 8.0^{\ddagger}$ & $15.8 \pm 2.9^{\ddagger}$ & $35.6 \pm 10.0^{\ddagger}$ \\
Lipocytes & $29.0 \pm 4.9^{\ddagger}$ & $150.0 \pm 49.8^{\ddagger}$ & $120.0 \pm 39.0^{\ddagger}$ \\
Collagen IV & & & \\
Hepatocytes & $1.0 \pm 0^{*}$ & $1.7 \pm 0.3$ & $0.9 \pm 0.0$ \\
Endothelial cells & $99.4 \pm 20.6^{\ddagger}$ & $38.5 \pm 5.4^{\ddagger}$ & $40.0 \pm 2.6^{\ddagger}$ \\
Lipocytes & $35.7 \pm 4.2^{\ddagger}$ & $99.3 \pm 19.4^{\ddagger}$ & $66.8 \pm 10.4^{\ddagger}$ \\
Laminin & & & \\
Hepatocytes & $1.0 \pm 0^{*}$ & $1.7 \pm 0.4$ & $1.0 \pm 0.1$ \\
Endothelial cells & $13.1 \pm 8.4^{\ddagger}$ & $9.6 \pm 3.1^{\ddagger}$ & $16.2 \pm 3.7^{\ddagger}$ \\
Lipocytes & $5.8 \pm 2.2^{\ddagger}$ & $13.6 \pm 4.3^{\ddagger}$ & $10.1 \pm 4.2^{\ddagger}$ \\
& & & \\
\hline
\end{tabular}

* All data are expressed as relative values, in comparison to normal hepatocytes (arbitrarily set at 1.0 ). Numbers represent mean \pm SEM for three separate animals. ${ }^{\ddagger} P<0.05$ vs. normal hepatocytes, by Mann-Whitney U-test. 

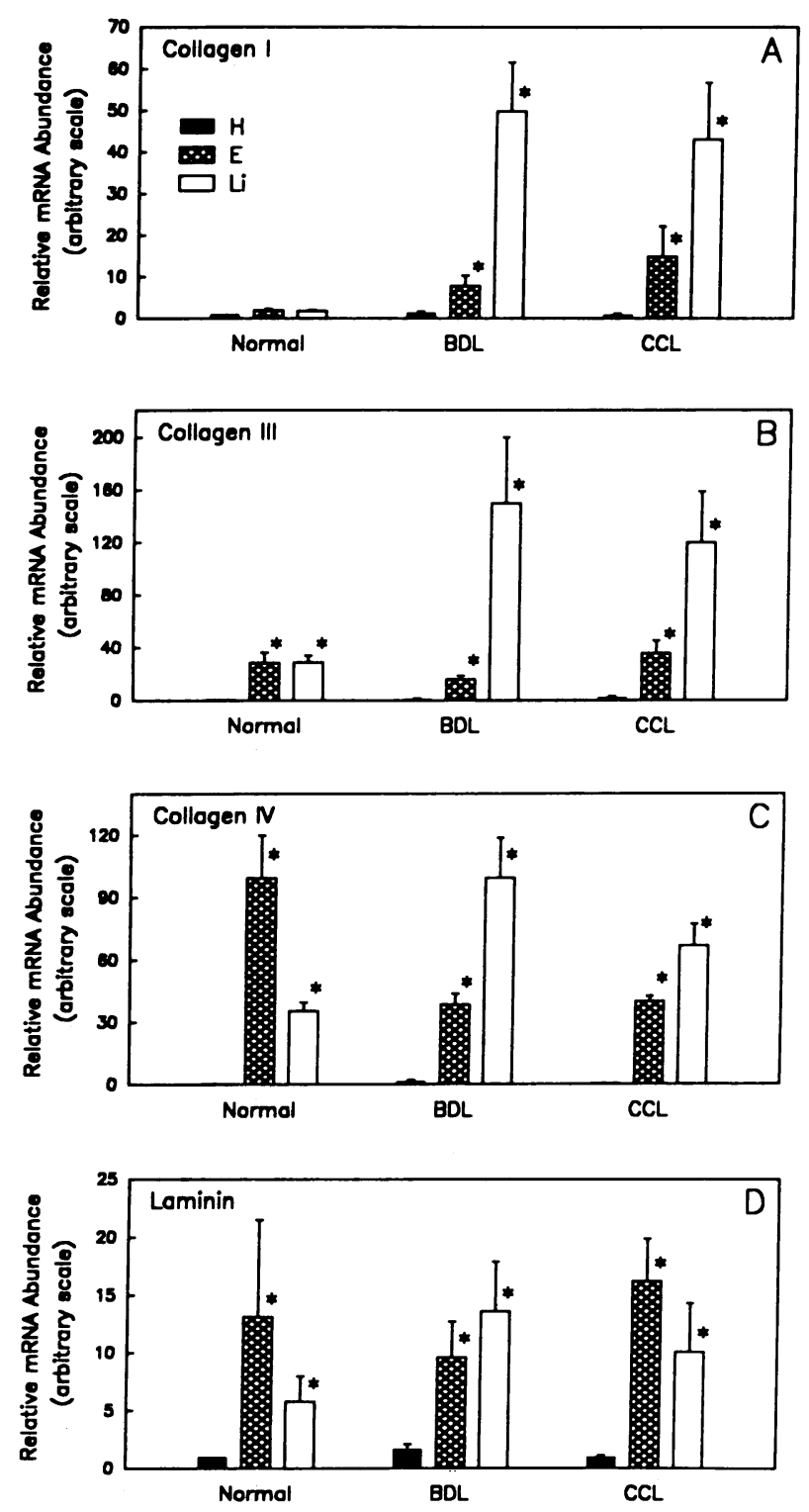

Figure 4. Steady-state levels of mRNA for four extracellular matrix genes in cells from normal and fibrotic liver. Histograms illustrate the numerical data in Table II for type I collagen $(A)$, type III collagen $(B)$, type IV collagen $(C)$, and laminin $(D)$. Note that the ordinate in each graph is different, making the results for hepatocytes in panels $B$ and $C$ appear indistinct. Bars represent mean \pm SEM for hepatocytes $\square$, endothelial cells $\square$, and lipocytes $\square$ from three separate animals. ${ }^{*} P<0.05$ vs. normal hepatocytes.
Table III. Steady-state Levels of $\beta$-Actin mRNA in Cells from Normal and Injured Rat Liver

\begin{tabular}{llrr}
\hline & Normal & \multicolumn{1}{c}{ BDL* } & \multicolumn{1}{c}{$\mathrm{CCL}^{*}$} \\
\hline Hepatocytes & $1.0 \pm 0^{\ddagger}$ & $1.4 \pm 0.3$ & $1.0 \pm 0.1$ \\
Endothelial cells & $8.4 \pm 2.1$ & $11.3 \pm 2.0$ & $5.6 \pm 1.1$ \\
Lipocytes & $3.1 \pm 0.5$ & $3.2 \pm 0.4$ & $2.0 \pm 0.6$
\end{tabular}

* $P>0.05$ for each cell population from BDL and CCL liver, in comparison to the same cell population from normal liver. ${ }^{\ddagger}$ All data are expressed as relative values, in comparison to normal hepatocytes (arbitrarily set at 1.0). Numbers represent mean \pm SEM for three separate animals.

Beta-actin mRNA was also quantitated in each of the cell populations (Table III; Fig. 6). The amount of total RNA that was specific for $\beta$-actin was not constant among the three cell types: relative proportions of $\beta$-actin mRNA were $1: 8: 3$ in hepatocytes, sinusoidal endothelia, and lipocytes, respectively. Steady-state levels of $\beta$-actin mRNA did not change in any of the cell types after experimental liver injury, indicating that the alterations noted in extracellular matrix gene expression were selective. Even when normalized for $\beta$-actin, mRNA levels for types I and III collagen were significantly increased in lipocytes from injured livers (data not shown).

\section{Discussion}

The preceding experiments demonstrate that collagen gene expression, particularly that for interstitial collagen, increases dramatically in liver following a fibrogenic stimulus. Furthermore, enhancement of interstitial collagen gene expression is most striking in a single population of liver cells, namely hepatic lipocytes. After experimental liver injury, mRNA levels for type I collagen rise to over 30 times those in normal lipocytes; type III collagen gene expression is increased to approximately 5 times over normal. Smaller, albeit significant, changes are observed in sinusoidal endothelial cells with respect to type I collagen.

The cellular source of extracellular matrix proteins and their mRNAs in liver has been the subject of some debate. Messenger RNAs for collagen types I, III, and IV, and laminin have been localized by in situ hybridization to sinusoidal cells in fibrotic liver (11-13); their precise cellular distribution, though, cannot be distinguished at the level of light microscopy. By measuring extracellular matrix gene expression in

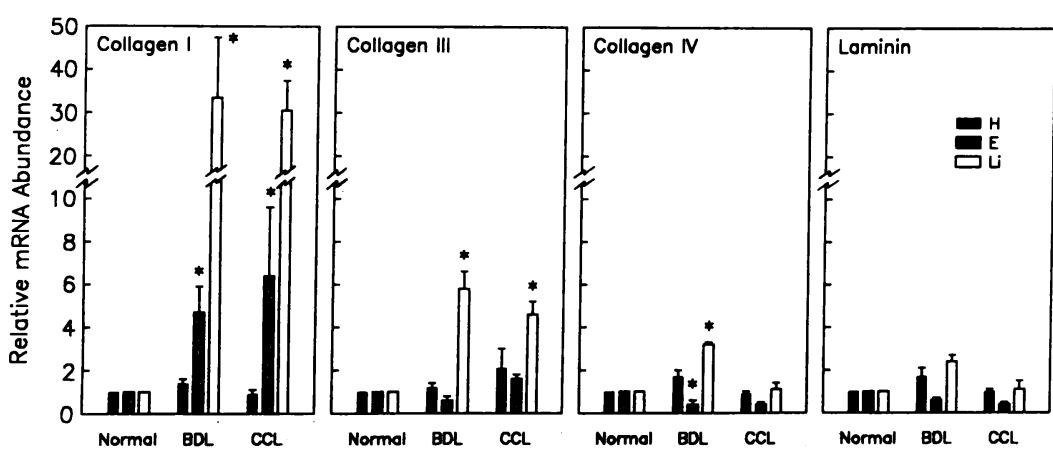

Figure 5. Cell-specific increase in extracellular matrix gene expression during fibrogenesis. Graphs represent specific matrix mRNA abundance in cells from BDL and CCL-treated liver, relative to the same cells in normal liver. Values for normal liver cells are arbitrarily set at 1.0 . Bars represent mean \pm SEM for hepatocytes $₫$, endothelial cells $\square$, and lipocytes $\square$ from three separate animals. ${ }^{*} P<0.05$ vs. normal liver cells. 


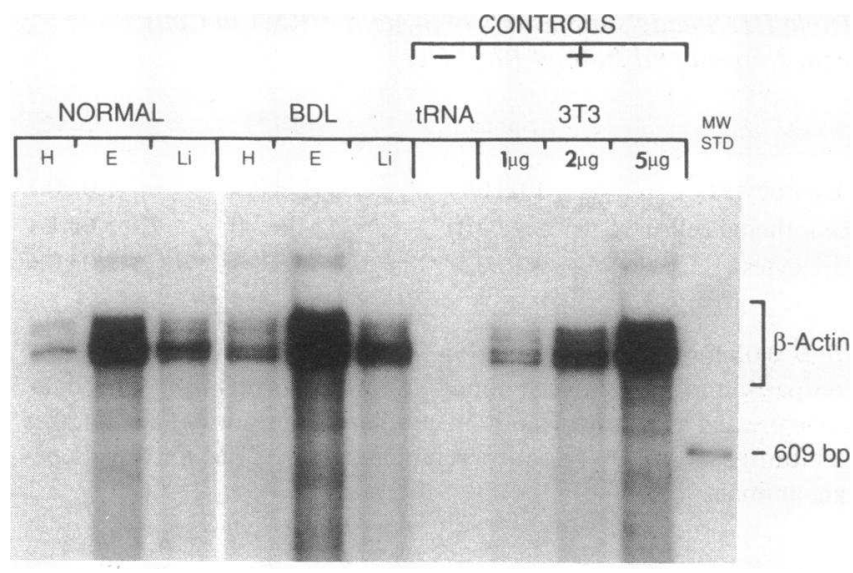

Figure 6. Actin mRNA in liver cells. Autoradiogram illustrating steady-state levels of $\beta$-actin mRNA in cells isolated from normal and fibrotic liver. Abbreviations as in Fig. 2. Actin signals are strongest in endothelial cells, followed by lipocytes and hepatocytes. This relationship does not change after either bile duct ligation $(B D L)$ or carbon tetrachloride administration (not shown).

purified liver cell isolates, we have been able not only to localize collagen mRNA to specific populations of liver cells, but also to quantitate the relative amount of collagen mRNA in each cell type. Furthermore, because all studies were performed on cells immediately following isolation, the data are relevant to the liver in vivo. The pronounced increase in types I and III collagen mRNA in the lipocyte population assigns a central role to these cells in the fibrotic process in vivo.

Extracellular matrix gene expression increases rapidly in lipocytes after a fibrogenic stimulus. Carbon tetrachloride administration promotes a marked increase in interstitial collagen gene expression by day 14 , before the onset of histologic cirrhosis. Bile duct ligation results in a similar change after only $5 \mathrm{~d}$, but substantial connective tissue deposition is already apparent histologically, preventing a firm conclusion regarding the time course of extracellular matrix gene expression with respect to matrix protein production in this model. That fibrosis is preceded by an increase in collagen mRNA is suggested by other studies, which demonstrate that type I collagen gene expression in whole liver rises within $24 \mathrm{~h}$ after bile duct ligation (32).

Both bile duct ligation and carbon tetrachloride administration, two mechanistically distinct types of liver injury, produce similar changes in matrix gene expression by mesenchymal liver cells. Bile duct ligation results in connective tissue deposition around portal triads with a paucity of associated inflammation or necrosis. Carbon tetrachloride, on the other hand, produces a pericentral pattern of fibrosis, with antecedent parenchymal injury. Small quantitative differences were observed in steady-state levels of collagen mRNA in obstructive as compared to toxin-induced fibrosis (Fig. 4); these probably reflect the more rapid time course of fibrosis in the former $(33,34)$. Apart from the quantitative results, the pattern of type I collagen gene expression in both models suggests that mesenchymal cell activation is a central pathogenic event in both types of fibrotic liver injury.

Sinusoidal endothelial cells as well as lipocytes exhibit significant increases in type I collagen mRNA after experimental liver injury. This is not surprising, in view of evidence that endothelial cells from other organs are active in matrix synthesis $(35,36)$. It is possible that some of the type I collagen mRNA measured in endothelial cells derives from contaminating lipocytes; if so, however, similar increases would be expected for collagen types III and IV and laminin. The results indicate that mRNAs for the latter three matrix proteins do not modulate in parallel in endothelial cells and lipocytes; in fact, type IV collagen mRNA levels vary inversely in the two populations during fibrogenesis. Thus, endothelial cells themselves must not be ignored as potential contributors to hepatic fibrosis in vivo.

In neither model of hepatic fibrogenesis is extracellular matrix gene expression modulated in hepatocytes. Indeed, mRNA for all collagen types is barely detectable in hepatocytes from either normal or injured liver, suggesting that parenchymal cells are minimally active in collagen synthesis. This conclusion contradicts that of Brenner and colleagues (37), who have recently reported that hepatocytes from normal rats contain up to three times as much type I collagen mRNA as nonparenchymal cells. The latter investigators prepared nonparenchymal cells by collagenase perfusion and centrifugal elutration, under conditions that selectively remove both lipocytes and sinusoidal endothelial cells. The remaining nonparenchymal fraction was presumably rich in Kupffer cells, which would not be expected to contain type I collagen mRNA. Hepatocytes in their study did exhibit strong signals for type I collagen mRNA; their methods, though, do not permit a rigid comparison of collagen gene expression between hepatocytes and endothelial cells or lipocytes. Earlier studies from this group suggested that parenchymal cells produce the bulk of hepatic collagen in vivo, both in normal and carbon tetrachloride-treated rats $(10,38)$. These conclusions were based on in vivo labeling of newly synthesized collagen, followed by determination of the cellular source of the protein upon extraction from the intact organ. Solubilization and recovery of collagen from whole liver, however, is not quantitative, raising the possibility that the collagen analyzed in these studies was not representative of total hepatic collagen. Our experiments confirm the results of in situ hybridization studies, in which nonparenchymal cells contribute most significantly to hepatic fibrosis in vivo.

The only extracellular matrix mRNA readily detected in hepatocytes in our experiments was that for laminin. Identification of laminin mRNA in these cells is unlikely to reflect contamination of the population by nonparenchymal cells, as hepatocyte isolates prepared by centrifugal elutriation are $>98 \%$ pure (Table I). No change in laminin mRNA was noted in hepatocytes after experimental liver injury; in view of the small changes in laminin gene expression in all liver cells, however, the relevance of this finding to the intact liver remains to be clarified (see below).

Type IV collagen and laminin gene expression were not significantly enhanced in the current experiments, in any of the cell types examined. These results are unexplained, in view of previous data indicating an increase in both type IV collagen and laminin during hepatic fibrosis in vivo (4). It is possible that enhanced translation of existing type IV and laminin mRNA account for such an increase; alternatively, alterations in type IV collagen and laminin mRNA may be delayed relative to collagen types I and III, and thus not observed during early fibrogenesis as exemplified in the current experiments. Pierce and colleagues (39) have found that in whole liver, type 
IV collagen gene expression does not increase significantly for 5 wk following carbon tetrachloride administration. After bile duct ligation, however, type IV collagen and laminin mRNA are increased fourfold within $5 \mathrm{~d}$ (32). This suggests that biliary ductular cells, which proliferate rapidly following bile duct ligation, may contribute to the increase in this model of fibrosis. Milani and colleagues (13) have recently shown by in situ hybridization that type IV collagen mRNA is located in the vicinity of biliary epithelial cells in experimental biliary fibrosis.

Based on the work of Clayton and Darnell (20), who demonstrated that specific cellular mRNAs are preserved in hepatocytes following collagenase perfusion, we are confident that our assessment of extracellular matrix gene expression in these cells is representative of that in vivo. While selective degradation of mRNA may occur in nonparenchymal cells during isolation, such a loss would result in underestimation of extracellular matrix gene expression by endothelial cells and lipocytes. Given that the existing results already demonstrate a significant difference between hepatocytes and nonparenchymal cells, this concern does not affect our overall conclusions. The quantitative measurements of extracellular matrix gene expression in parenchymal and nonparenchymal cells (Fig. 4) suggest that extracellular matrix mRNAs are more abundant in lipocytes and endothelial cells than in hepatocytes. This is true for types III and IV collagen and laminin in normal liver, and for all the matrix genes examined during fibrogenesis. These comparisons assume that hepatocytes, sinusoidal endothelial cells, and lipocytes contain similar amounts of total cellular RNA. In the event that total RNA content is different in each cell type, our results cannot be used to interpret the relative contribution of each cell type to hepatic fibrosis on a per-cell basis. Studies are currently underway to clarify this issue. Based on RNA/DNA ratios measured in hepatocytes (40) and mesenchymal cells (41), the total RNA content of lipocytes is estimated at $42 \%$ of that in hepatocytes. Taking into account this difference, the increase in interstitial collagen gene expression in lipocytes still far exceeds that in hepatocytes. Thus, lipocytes, and to a lesser extent endothelial cells, appear to be the main contributors to hepatic fibrosis in vivo.

Extracellular matrix protein production was not measured in parallel with collagen gene expression in these experiments. Studies of lipocytes in culture, however, demonstrate that increases in collagen mRNA are associated with increased collagen synthesis (Maher, J. J., unpublished observations). Although it cannot be excluded that posttranscriptional events control synthesis of extracellular matrix by lipocytes in vivo, it is unlikely that their contribution to fibrosis is overshadowed by that of hepatocytes, which contain little or no matrix-specific $\mathrm{mRNA}$.

It is of interest that in normal liver, both parenchymal and nonparenchymal cells contain very low levels of type I collagen mRNA. Even using a sensitive assay (RNase protection), type I collagen mRNA is difficult to identify in any of the cell types examined. This supports the concept that in normal adult liver, synthesis of type I collagen is minimally active. Both bile duct ligation and carbon tetrachloride administration alter this state. Although the mechanism whereby matrix synthesis is activated is unknown, our studies suggest that these different modes of injury elicit a similar response and thus may act through common signals. These could include humoral factors, such as cytokines elaborated by recruited macrophages or resident liver cells (42-44), or stimuli in the form of altered cell-matrix interactions, provoked by local release of matrix-degrading proteinases during liver injury (45). Exploration of these possibilities is currently underway, using the approach outlined here.

\section{Acknowledgments}

Expert technical assistance was provided by Melanie Mark, Glenn Yamasaki, and John Niemiec.

This work was supported by U.S. Public Health Service grants from the National Institute of Alcohol Abuse and Alcoholism (AA-07810) and the National Institutes of Health (DK-31198, 1P-30 DK-26743, and T32 DK-07007).

\section{References}

1. Rojkind, M., M. A. Giambrone, and L. Biempica. 1979. Collagen types in normal and cirrhotic liver. Gastroenterology. 76:710-719.

2. Murata, K., M. Kudo, R. Onuma, and T. Motoyama. 1984. Changes of collagen types at various stages of human liver cirrhosis. Hepato-Gastroenterology. 31:158-161.

3. Seyer, J. M., E. T. Hutcheson, and A. H. Kang. 1977. Collagen polymorphism in normal and cirrhotic human liver. J. Clin. Invest. 59:241-248

4. Hahn, E., G. Wick, D. Pencev, and R. Timpl. 1980. Distribution of basement membrane proteins in normal and fibrotic human liver: collagen type IV, laminin, and fibronectin. Gut. 21:63-71.

5. Martinez-Hernandez, A. 1984. The hepatic extracellular matrix. I. Electron immunohistochemical studies in normal rat liver. $L a b$. Invest. 51:57-74.

6. Clement, B., H. Emonard, M. Rissel, M. Druguet, J.-A. Grimaud, D. Herbage, M. Bourel, and A. Guillouzo. 1984. Cellular origin of collagen and fibronectin in the liver. Cell. Mol. Biol. 30:489-496.

7. Clement, B., M. Rissel, S. Peyrol, Y. Mazurier, J.-A. Grimaud, and A. Guillouzo. 1985. A procedure for light and electron microscopic intracellular immunolocalization of collagen and fibronectin in rat liver. J. Histochem. Cytochem. 33:407-414.

8. Sakakibara, K., S. Igarashi, and T. Hatahara. 1985. Localization of type III procollagen aminopeptide antigenicity in hepatocytes from cirrhotic human liver. Virchows Arch. A Pathol. Anat. Histol. 408:219-228.

9. Martinez-Hernandez, A. 1985. The hepatic extracellular matrix. II. Electron immunohistochemical studies in rats with $\mathrm{CCl}_{4}$-induced cirrhosis. Lab. Invest. 53:166-186.

10. Chojkier, M., K. D. Lyche, and M. Filip. 1988. Increased production of collagen in vivo by hepatocytes and nonparenchymal cells in rats with carbon tetrachloride-induced hepatic fibrosis. Hepatology (Baltimore). 8:803-814.

11. Milani, S., H. Herbst, D. Schuppan, E. G. Hahn, and H. Stein. 1989. In situ hybridization for procollagen types I, III and IV mRNA in normal and fibrotic rat liver: evidence for predominant expression in nonparenchymal liver cells. Hepatology (Baltimore). 10:84-92.

12. Milani, S., H. Herbst, D. Schuppan, E. O. Riecken, and H. Stein. 1989. Cellular localization of laminin gene transcripts in normal and fibrotic human liver. Am. J. Pathol. 134:1175-1182.

13. Milani, S., H. Herbst, D. Schuppan, K. Y. Kim, E. O. Riecken, and H. Stein. 1990. Procollagen expression by nonparenchymal rat liver cells in experimental biliary fibrosis. Gastroenterology. 98:175184.

14. Friedman, S. L., F. J. Roll, J. Boyles, and D. M. Bissell. 1985. Hepatic lipocytes: the principal collagen-producing cells of normal rat liver. Proc. Natl. Acad. Sci. USA. 82:8681-8685.

15. Senoo, H., R.-I. Hata, Y. Nagai, and K. Wake. 1984. Stellate cells (vitamin A-storing cells) are the primary site of collagen synthesis in nonparenchymal cells in the liver. Biomed. Res. 5:451-458.

16. Maher, J. J., D. M. Bissell, S. L. Friedman, and F. J. Roll. 1988. 
Collagen measured in primary cultures of normal rat hepatocytes derives from lipocytes within the monolayer. J. Clin. Invest. 82:450-459.

17. Friedman, S. L., F. J. Roll, J. Boyles, D. M. Arenson, and D. M. Bissell. 1989. Maintenance of differentiated phenotype of cultured rat hepatic lipocytes by basement membrane matrix. J. Biol. Chem. 264:10756-10762.

18. Davis, B. H., B. M. Pratt, and J. A. Madri. 1987. Retinol and extracellular collagen matrices modulate hepatic Ito cell collagen phenotype and cellular retinol binding protein levels. J. Biol. Chem. 262:10280-10286.

19. Weiner, F. R., M. A. Giambrone, M. J. Czaja, A. Shah, G. Annoni, S. Takahashi, M. Eghbali, and M. A. Zern. 1990. Ito-cell gene expression and collagen regulation. Hepatology (Baltimore). 11:111117.

20. Clayton, D. F., and J. E. Darnell. 1983. Changes in liver-specific compared to common gene transcription during primary culture of mouse hepatocytes. Mol. Cell. Biol. 3:1552-1561.

21. Irving, M. G., F. J. Roll, S. Huang, and D. M. Bissell. 1984. Characterization and culture of sinusoidal endothelium from normal rat liver: lipoprotein uptake and collagen phenotype. Gastroenterology. 87:1233-1247.

22. Friedman, S. L., and F. J. Roll. 1987. Isolation and culture of hepatic lipocytes, Kupffer cells, and sinusoidal endothelial cells by density gradient centrifugation with Stractan. Anal. Biochem. 161:207-218.

23. Maher, J. J., S. L. Friedman, F. J. Roll, and D. M. Bissell. 1988. Immunolocalization of laminin in normal rat liver and biosynthesis of laminin by hepatic lipocytes in primary culture. Gastroenterology. 94:1053-1062.

24. Genovese, C., D. Rowe, and B. Kream. 1984. Construction of DNA sequences complementary to rat $\alpha_{1}$ and $\alpha_{2}$ collagen mRNA and their use in studying the regulation of type I collagen synthesis by 1,25-dihydroxyvitamin D. Biochemistry. 23:6210-6216.

25. Kurkinen, M., M. R. Condon, B. Blumberg, D. P. Barlow, S. Quinones, J. Saus, and T. Pihlajaniemi. 1987. Extensive homology between the carboxyl-terminal peptides of mouse $\alpha_{1}(\mathrm{IV})$ and $\alpha_{2}$ (IV) collagen. J. Biol. Chem. 262:8496-8499.

26. Barlow, D. P., N. M. Green, M. Kurkinen, and B. L. M. Hogan. 1984. Sequencing of laminin B chain cDNA's reveals C-terminal regions of coiled-coil alpha-helix. EMBO (Eur. Mol. Biol. Organ.) J. 3:2355-2362.

27. Gunning, P., P. Ponte, H. Okayama, J. Engel, H. Blau, and L. Kedes. 1983. Isolation and characterization of full-length cDNA clones for human $\alpha$-, $\beta$-, and $\gamma$-actin mRNAs: skeletal but not cytoplasmic actins have an amino-terminal cysteine that is subsequently removed. Mol. Cell. Biol. 3:787-795.

28. Melton, D. A., P. A. Krieg, M. R. Rebagliati, T. Maniatis, K. Zinn, and M. R. Green. 1984. Efficient in vitro synthesis of biologically active RNA and RNA hybridization probes from plasmids containing a bacteriophage SP6 promoter. Nucleic Acids Res. 12:70357056.

29. Gilman, M. 1987. Ribonuclease protection assay. In Current Protocols in Molecular Biology. Vol. 1. F. M. Ausubel, R. Brent, R. E. Kingston, D. D. Moore, J. G. Seidman, J. A. Smith, and K. Struhl, editors. Greene Publishing Associates and Wiley Interscience, John Wiley and Sons, Inc., New York. 4.7.1-4.7.8.

30. Berger, S. L. 1987. Preparation and characterization of RNA: overview. Methods Enzymol. 152:215-227.

31. Mak, K. M., and C. S. Lieber. 1988. Lipocytes and transitional cells in alcoholic liver disease: a morphometric study. Hepatology (Baltimore). 8:1027-1033.

32. Maher, J. J. 1989. Rapid induction of extracellular matrix gene expression and collagen synthesis following bile duct ligation in the rat. Hepatology (Baltimore). 10:631. (Abstr.)

33. Kountouras, J., B. H. Billing, and P. J. Scheuer. 1984. Prolonged bile duct obstruction: a new experimental model for cirrhosis in the rat. Br. J. Exp. Pathol. 65:305-311.

34. Perez-Tamayo, R. 1979. Cirrhosis of the liver: a reversible disease? Pathol. Annu. 14:183-213.

35. Madri, J. A., and S. K. Williams. 1983. Capillary endothelial cell cultures: phenotypic modulation by matrix components. J. Cell Biol. 97:153-165.

36. Tseng, S. C. G., N. Savion, D. Gospodarowicz, and R. Stern. 1983. Modulation of collagen synthesis by a growth factor and by the extracellular matrix: comparison of cellular response to two different stimuli. J. Cell Biol. 97:803-809.

37. Brenner, D. A., J. M. Alcorn, S. P. Feitelberg, H. L. Leffert, and M. Chojkier. 1990. Expression of collagen genes in liver. Mol. Biol. \& Med. 7:105-115.

38. Chojkier, M. 1986. Hepatocyte collagen production in vivo in normal rats. J. Clin. Invest. 78:333-339.

39. Pierce, R. A., M. R. Glaug, R. S. Greco, J. W. Mackenzie, C. D. Boyd, and S. B. Deak. 1987. Increased procollagen mRNA levels in carbon tetrachloride-induced liver fibrosis in rats. J. Biol. Chem. 262:1652-1658.

40. Flaim, K. E., S. M. Hutson, C. E. Lloyd, J. M. Taylor, R. Shiman, and L. S. Jefferson. 1985. Direct effect of insulin on albumin gene expression in primary cultures of rat hepatocytes. Am. J. Physiol. 249:E447-E453.

41. Tolstoshev, P., R. Haber, B. C. Trapnell, and R. G. Crystal. 1981. Procollagen messenger RNA levels and activity and collagen synthesis during the fetal development of sheep lung, tendon, and skin. J. Biol. Chem. 256:9672-9679.

42. Friedman, S. L., and M. J. P. Arthur. 1989. Activation of cultured rat hepatic lipocytes by Kupffer cell conditioned medium. $J$. Clin. Invest. 84:1780-1785.

43. Czaja, M. J., F. R. Weiner, K. C. Flanders, M. A. Giambrone, R. Wind, L. Biempica, and M. A. Zern. 1989. The in vitro and in vivo association of transforming growth factor- $\beta 1$ with hepatic fibrosis. $J$. Cell Biol. 108:2477-2482.

44. Weiner, F. R., A. Shah, L. Biempica, M. A. Zern, and M. J. Czaja. 1989. Cellular sources of increased collagen and transforming growth factor- $\beta 1$ gene expression in fibrotic rat liver. Hepatology (Baltimore). 10:629. (Abstr.)

45. Arthur, M. J. P., S. L. Friedman, F. J. Roll, and D. M. Bissell. 1989. Lipocytes from normal rat liver release a neutral metalloproteinase that degrades basement membrane (type IV) collagen. J. Clin. Invest. 84:1076-1085. 